\title{
Estruturas, Modelos E os Fundamentos Da Abordagem Semântica
}

\author{
Jonas R. BECKer ARENHART \\ FERNANDO T. F. MORAES \\ Universidade Federal de Santa Catarina
}

\begin{abstract}
In this paper, gathering several topics present in the work of Newton da Costa, we propose a rigorous foundation for a possible formulation of scientific theories according to the semantic approach. Following da Costa, as a first step we develop a general theory of structures; inside this theory we show how we can characterize formal languages as particular kinds of structures, more specifically, as free algebras. Next we discuss how we can link a language to a structure, with which we can formulate the axioms that are intended to capture the theory of the structure. Finally, we show how we can, employing the framework developed, formulate da Costa and Chaqui's formalization of the so-called Suppes' Predicate, used to characterize scientific theories in a rigorous way.
\end{abstract}

Keywords: Semantic approach, structures, models, Suppes' predicate.

Dedicado ao Prof. Newton da Costa pelos seus 80 anos.

\section{Introdução}

Um dos grandes desafios para a filosofia da ciência no século XX é responder à pergunta: o que é uma teoria científica? Esta pergunta é particularmente importante pois é com relação à teorias que formamos nossas crenças; as teorias são aquilo que aceitamos ou rejeitamos; e são as teorias que, de alguma forma, se relacionam com o mundo, e permitem o progresso em nosso conhecimento. Então, especificar em que consiste uma teoria científica é tarefa de grande relevância para a filosofia da ciência. A história recente das disputas acerca de uma resposta adequada a esta questão, conforme ela nos é contada pelos adeptos da visão atualmente predominante, nos diz que temos duas alternativas: a abordagem sintática e a abordagem semântica. Comecemos com um breve esboço da abordagem sintática, já que ela surgiu primeiro e a abordagem semântica foi formulada como uma reação às suas alegadas limitações.

O problema acerca da natureza das teorias científicas teve grande destaque nos trabalhos de um grupo de filósofos que se denominavam o Círculo de Viena, que passaram a se reunir em Viena sob a liderança de Moritz Schlick no começo da década de 20, e que dominou a cena filosófica por várias décadas. Os membros do Círculo eram

Principia 14(1): 15-30 (2010).

Published by NEL — Epistemology and Logic Research Group, Federal University of Santa Catarina (UFSC), Brazil. 
em sua maioria filósofos e cientistas interessados em dar uma fundamentação científica à filosofia, contra os excessos da filosofia idealista do final do séc.XIX, adotando uma forma de empirismo que buscaram tornar compatível com a ciência da época. A abordagem do Círculo ficou conhecida como Received View, ou abordagem sintática. ${ }^{1}$ Segundo esta concepção, uma teoria é constituída de: i) um cálculo lógico abstrato; ii) um conjunto de fórmulas deste cálculo, os axiomas da teoria; iii) um conjunto de regras de correspondência. O cálculo lógico, além de um aparato dedutivo, tem seu vocabulário não-lógico dividido em duas partes: os termos observacionais e os termos teóricos. As regras de correspondência relacionam estes termos, nos mostrando como entender os termos teóricos em função dos termos observacionais. Postulados teóricos então são formulados utilizando-se apenas o vocabulário teórico que, junto com as regras de correspondência, permitem que se produza uma interpretação da teoria. Muitas dificuldades foram apontadas relativamente a esta abordagem, dentre elas estavam sua extrema artificialidade e o fato de que diferentes formulações de uma mesma teoria, por utilizar por exemplo um vocabulário diferente, ou introduzir novas regras de correspondência, deveriam contar como teorias diferentes, algo que não parece razoável para nossa compreensão intuitiva de teorias (a história e análise detalhada dessa concepção pode ser encontrada em Suppe 1977).

Contra este modo de ver as teorias surgiu uma nova abordagem a partir da década de 50, tendo como um de seus proponentes P. Suppes, e amplamente adotada atualmente por filósofos de diferentes escolas, tanto realistas quanto anti-realistas. Segundo uma das formas desta abordagem, chamada de abordagem semântica às teorias científicas (para um excelente resumo histórico dessa abordagem, ver Suppe 1989), grosso modo, para caracterizarmos uma teoria não devemos nos concentrar na particular linguagem que utilizamos para formulá-la, mas antes nos modelos dos axiomas. Assim, a formulação dos axiomas determina uma classe de modelos, e mesmo que diferentes formulações possam caracterizar uma mesma classe, nada será perdido pois a ênfase é dada às estruturas designadas, não ao modo como elas são selecionadas. Com isto, alguns defensores da abordagem semântica chegam ao ponto de afirmar que teorias são entidades extra-linguísticas, no sentido de não dependerem da particular linguagem utilizada para selecionar a classe de modelos que caracteriza a teoria. Esta ênfase nos modelos, que caracteriza a abordagem semântica, suscita também muitas dúvidas. O que são modelos? Modelos em que sentido desta palavra? Que tipo de aparato conceitual é empregado para apresentar os modelos? Os modelos utilizados pela abordagem semântica podem ser empregados para esclarecer diferentes aspectos da prática científica?

Neste artigo, trataremos de algumas destas questões concernentes à abordagem semântica. Se devemos ser rigorosos, então os detalhes devem ser apresentados e as lacunas preenchidas. Primeiramente, nos restringiremos a um sentido bastante preciso do termo modelo: o sentido lógico, modelo como uma estrutura conjuntista. 
Neste sentido, para que uma estrutura matemática seja um modelo, devemos especificar um conjunto de fórmulas da qual ela é um modelo. Assim, apresentar uma teoria científica, mesmo na abordagem semântica, envolve o uso de linguagens, malgrado alguns defensores desta concepção. Como fazemos a ligação entre a linguagem e as estruturas que caracterizam a teoria? Como veremos, podemos fazer isto através do famoso predicado de Suppes, uma fórmula da linguagem da teoria de conjuntos que serve para relacionar estruturas com os axiomas dos quais eles são modelos.

Nosso objetivo é tratar destes temas fazendo uma conexão entre várias propostas presentes na obra de Newton C. A. da Costa, que em vários trabalhos, em colaboração com filósofos e matemáticos, propôs as peças chave para uma forma de compreender o problema. Aqui, faremos a ligação entre os elementos presentes na obra de da Costa e daremos a eles a coerência necessária para que uma resposta rigorosa ao problema em questão seja estabelecida. Começaremos apresentando uma teoria geral de estruturas matemáticas. No contexto destas estruturas, podemos selecionar algumas delas, as álgebras livres, que irão desempenhar o papel de linguagens formais. Discutiremos a relação entre linguagem e estruturas, especificando como se pode estabelecer uma linguagem que "fala" de modo natural dos elementos e relações em uma estrutura, sendo assim conveniente para que se formule os axiomas de uma teoria da qual a estrutura em questão é um dos modelos. Na última etapa, generalizamos a abordagem para definir uma classe de estruturas, que serão modelos de um certo conjunto de axiomas, definindo um predicado de Suppes, conforme formalizado no trabalho de da Costa e Chuaqui (ver da Costa e Chuaqui 1988).

Passemos primeiramente às estruturas.

\section{Uma teoria de estruturas}

A noção de estrutura desempenha há muito um papel importante na matemática clássica, papel que se tornou central desde que o coletivo francês Bourbaki, em meados do século passado, buscou reescrever a matemática evidenciando seu caráter estrutural. Para Bourbaki, a matemática pode ser vista como o estudo das estruturas mães (algébricas, de ordem e topológicas), e das estruturas oriundas da combinação destas (Bourbaki 1948, 1968). Nesta seção, seguiremos a apresentação de da Costa, Rodrigues 2007, fazendo pequenas modificações que ficarão patentes no decorrer do texto. Todos os desenvolvimentos serão realizados em ZFC informal. Primeiramente, devemos observar que todo o trabalho aqui desenvolvido pode, em princípio, ser feito em teoria de categorias. Nossa opção por ZFC reflete tanto o fato de ser a ferramenta mais comumente utilizada pelos filósofos da ciência (mesmo que de modo implícito) como por haver sido eleita por P. Suppes para suas investigações em filosofia da ciência. 
Nossa primeira definição será do conjunto $\tau$ de tipos. Esse conjunto será também importante no desenvolvimento das linguagens formais na próxima seção.

Definição 2.1 O conjunto $\tau$ de tipos é o menor conjunto satisfazendo as seguintes condições:

1. Os símbolos $0,1, \ldots n-1$, com $1 \leq n<\omega$, pertencem a $\tau$;

2. Se $a_{0}, a_{1}, \ldots, a_{n-1} \in \tau$, então $\left\langle a_{0}, a_{1}, \ldots, a_{n-1}\right\rangle \in \tau, 1 \leq n<\omega$, onde $\left\langle a_{0}, a_{1}\right.$, $\left.\ldots, a_{n-1}\right\rangle$ é uma sequência finita de $n$ termos, composta por $a_{0}, a_{1}, \ldots, a_{n-1}$.

Definiremos agora a ordem de um elemento de $\tau$.

Definição 2.2 Se $a \in \tau$, a ordem de a, denotada por ord(a), é definida como:

1. $\operatorname{ord}(k)=0$, para $k=0,1, \ldots, n-1$;

2. $\operatorname{ord}\left(\left\langle a_{0}, a_{1}, \ldots, a_{n-1}\right\rangle\right)=\max \left\{\operatorname{ord}\left(a_{0}\right), \operatorname{ord}\left(a_{1}\right), \ldots, \operatorname{ord}\left(a_{n-1}\right)\right\}+1$.

Ou seja, ord é uma função do conjunto $\tau$ no conjunto $\omega$ dos números naturais.

A partir de uma família de conjuntos base $D_{0}, D_{1}, \ldots, D_{n-1}$, os conjuntos domínio da estrutura, a definição seguinte nos permite obter as relações e propriedades sobre essa família. Nesta definição, as operações conjuntistas usuais de conjunto potência e produto cartesiano são usadas; serão denotadas, respectivamente, por "भுP" e " $\times$ ".

Definição 2.3 Seja $D_{0}, D_{1}, \ldots, D_{n-1}$ uma família de conjuntos não vazios. Definimos uma função $t$, chamada de escala baseada em $D_{0}, D_{1}, \ldots, D_{n-1}$ tendo $\tau$ como domínio da seguinte forma:

1. $t(k)=D_{k}$, para $k=0,1, \ldots, n-1$;

2. Se $a_{0}, a_{1}, \ldots, a_{n-1} \in \tau$, então $t\left(\left\langle a_{0}, a_{1}, \ldots, a_{n-1}\right\rangle\right)=\mathscr{P}\left(t\left(a_{0}\right) \times t\left(a_{1}\right) \times \ldots \times\right.$ $\left.t\left(a_{n-1}\right)\right)$.

Através da função $t$, intuitivamente falando, podemos construir relações e propriedades baseadas nos tipos, tendo como base os elementos da família $D_{n}$, isto é, $D_{0}, D_{1}, \ldots, D_{n-1}$. Dado $a \in \tau$, os elementos de $t(a)$ são ditos do tipo $a$. Conforme a ordem dos tipos aumenta, cresce também a complexidade dos objetos atribuídos a eles. Os elementos de tipo $k=0,1, \ldots, n-1$, por exemplo, (isto é, elementos de $D_{k}$, pela definição acima) são chamados indivíduos de tipo $k$, para cada $0 \leq k \leq n-1$. Os elementos de $t(\langle\langle 1\rangle\rangle)$ são propriedades de propriedades de indivíduos de tipo 1 , como podemos ver pela definição acima. Como outro exemplo, os elementos de $t(\langle 0,0,1\rangle)$ são relações ternárias nas quais os primeiros dois relata são indivíduos 
de tipo 0 e o terceiro é de tipo 1 ; já os elementos de $t(\langle\langle 0,1\rangle, 1\rangle)$ são relações; elas relacionam uma relação entre indivíduos de tipos 0 e 1 e um objeto de tipo 1 .

O conjunto $\bigcup\left(\operatorname{range}\left(D_{n}\right)\right)$ é chamado de escala baseada na família $D_{n}$, e o denotaremos por $\varepsilon\left(D_{n}\right)$.

Uma sequência é uma função cujo domínio é um número ordinal, finito ou infinito. Em nosso tratamento, família terá o mesmo sentido que sequência. A sequência $a_{0}, a_{1}, \ldots$ cujo domínio é o ordinal $\lambda$, será escrita $\left(a_{\iota}\right)_{\iota \in \lambda}$ ou $a_{\iota}, \iota \in \lambda$, ou simplesmente $a_{\iota}$, se o domínio estiver claro pelo contexto.

Definição 2.4 O cardinal $K_{D_{n}}$ associado a $\varepsilon\left(D_{n}\right)$ é definido como

$$
K_{D_{n}}=\sup \left\{\left|\bigcup_{k=0}^{n-1} D_{k}\right|,\left|\mathscr{P}\left(\bigcup_{k=0}^{n-1} D_{k}\right)\right|,\left|\mathscr{P}^{2}\left(\bigcup_{k=0}^{n-1} D_{k}\right)\right|, \ldots\right\}
$$

Aqui, $\left|\bigcup_{k=0}^{n-1} D_{k}\right|$ denota o cardinal do conjunto $\bigcup_{k=0}^{n-1} D_{k}$.

Definiremos agora a estrutura e sobre a família $D_{n}$.

Definição 2.5 Uma estrutura e baseada na família $D_{n}$ é um par ordenado da forma

$$
e=\left\langle D_{n}, R_{\iota}\right\rangle
$$

Aqui, $R_{\iota}$ é uma sequência de elementos de $\varepsilon\left(D_{n}\right)$, e supomos que o domínio dessa sequência é estritamente menor que $K_{D_{n}}$. Dizemos que $K_{D_{n}}$ e $\varepsilon\left(D_{n}\right)$ são o cardinal e a escala associados a $e$, respectivamente.

Como já dito, cada elemento de $\varepsilon\left(D_{n}\right)$ possui um certo tipo. A ordem de uma relação é definida como a ordem de seu tipo, ou seja, a ordem do tipo dos elementos que formam a relação. A ordem de $e$, denotada $\operatorname{ord}(e)$, é a ordem do maior dos tipos das relações da família $R_{\iota}$, se houver alguma, caso contrário, ord $(e)=\omega$.

As estruturas da matemática clássica podem todas ser reduzidas a estruturas de acordo com a definição apresentada. No início desta seção, assinalamos que a nossa apresentação conteria algumas modificações com relação a proposta original de da Costa e Rodrigues 2007. Divergimos ao permitir que indivíduos e operações ocorram nas estruturas, ao passo que da Costa e Rodrigues reduzem operações a relações e identificam indivíduos com seus conjuntos unitários. A principal razão dessa mudança é simplificar a exposição neste trabalho, já que do ponto de vista matemático essas diferenças são apenas uma questão de convenção. Portanto, na definição de estruturas aqui proposta os objetos da família $R_{\iota}$ podem ser não somente relações, mas operações também, isto é, relações satisfazendo a bem conhecida condição funcional, ou ainda elementos distinguidos do domínio. 


\section{Linguagens Formais}

Apresentaremos agora uma abordagem das linguagens formais desenvolvida a partir da noção da estrutura acima delineada. Aqui, linguagens serão uma tipo especial de estrutura, as álgebras livres. Nesta seção, utilizaremos o arcabouço da teoria de estruturas apresentada na seção anterior, e apresentaremos alguns tópicos de álgebra universal necessários a nossa exposição.

Definição 3.1 O tipo de similaridade de uma estrutura $e=\left\langle D, R_{\iota}\right\rangle$ é uma família $s_{\lambda<\iota}$ de tipos, tal que para cada $\lambda<\iota$, $s_{\lambda}$ é o tipo de $R_{\lambda}$.

Dizemos que duas estruturas têm o mesmo tipo de similaridade quando a família $s$ é igual em ambas, ou seja, quando suas relações têm o mesmo tipo. Como uma família é sempre ordenada, dadas duas estruturas com o mesmo tipo de similaridade, as relações em ambas ocorrem sempre na mesma ordem, a ordem dos tipos da família.

Definição 3.2 Uma s-álgebra $\mathfrak{A}$ é uma estrutura $\left\langle A, R_{\iota}\right\rangle$ tal que ord $\left(R_{n}\right) \leq 1$ para cada $n$ e $\mathfrak{A}$ tem tipo de similaridade $s$.

A restrição da ordem das relações para 1 ou menos serve para fazer cada $s$ álgebra uma álgebra no sentido usual, isto é, uma estrutura composta de um conjunto (o domínio) com uma família de operações definidas sobre esse conjunto.

Definição 3.3 Sejam $\mathfrak{A}, \mathfrak{B}$ s-álgebras com $\mathfrak{A}=\left\langle A, R_{\iota}\right\rangle$ e $\mathfrak{B}=\left\langle B, R_{\iota}^{\prime}\right\rangle$. Um homomorfismo de $\mathfrak{A}$ em $\mathfrak{B}$ é uma função $\varphi: A \rightarrow B$ tal que, para todo $R_{\lambda<\iota}$ da s-álgebra $\mathfrak{A}$ cujo tipo é $\left\langle 0_{1}, \ldots, 0_{k}\right\rangle$, temos:

$$
\varphi\left(R_{\lambda<\iota}\left(a_{1}, \ldots, a_{k}\right)\right)=R_{\lambda<\iota}^{\prime}\left(\varphi\left(a_{1}\right), \ldots, \varphi\left(a_{k}\right)\right) .
$$

Definição 3.4 Sejam $\mathfrak{A}$ e $\mathfrak{B}$ s-álgebras, e $\varphi: A \rightarrow B$ um homomorfismo. Se $\varphi$ é uma bijeção dizemos que a função $\varphi: A \rightarrow B$ é um isomorfismo entre $\mathfrak{A}$ e $\mathfrak{B}$

Definição 3.5 Seja X um conjunto, seja $\mathfrak{F}$ uma s-álgebra com domínio $F$ e seja $\sigma$ : $X \rightarrow F$ uma função. Dizemos que $\langle F, \sigma\rangle$ (também denotado por $F$ ) é uma s-álgebra livre sobre o conjunto $X$ de livre geradores se, para toda s-álgebra $\mathfrak{A}$ e função $\tau: X \rightarrow A$, existe um único homomorfismo $\varphi: F \rightarrow$ A tal que $\varphi \sigma=\tau$.

Teorema 3.1 Para todo conjunto $X$ e todo tipo de similaridade s, existe uma s-álgebra livre sobre $X$. Essa s-álgebra livre sobre $X$ é única a menos de isomorfismo.

A prova da existência de uma única álgebra livre para $X$ pode ser vista em Barnes e Mack 1975, com as devidas adaptações.

A partir dos desenvolvimentos acima realizados passamos, agora, ao desenvolvimento das linguagens formais como casos particulares de álgebras. 


\subsection{Uma linguagem formal para a teoria de tipos simples}

Apresentaremos nesta seção a linguagem da teoria de tipos simples como uma específica $s$-álgebra. Começamos com algumas definições.

Para cada tipo $a \in \tau$, temos:

1. Um conjunto enumerável $V_{a}$, dito o conjunto de variáveis de tipo $a$.

2. Um conjunto $R_{a}$ de símbolos de constante de tipo $a$, que eventualmente pode ser vazio para alguns elementos de $\tau$.

Definiremos agora o conjunto de todas as variáveis e constantes da linguagem.

Definição 3.6 Para todo $a \in \tau$ :

1. $V=\bigcup V_{a}$, o conjunto das variáveis;

2. $R=\bigcup R_{a}$, o conjunto das constantes.

Como podemos observar, para cada conjunto da família $D_{k}$ haverá uma família de variáveis restrita a $D_{k}$. Esta é, portanto, uma linguagem poli-sortida.

Para cada elemento de $\tau$, definimos um termo deste tipo do seguinte modo:

Definição 3.7 Seja $a \in \tau$, o conjunto $T$ de termos é definido por:

$$
T_{a}=V_{a} \cup R_{a} .
$$

O próximo passo consiste em especificar o conjunto de geradores para formar nossa álgebra livre. Vamos tomar $X$ como sendo o seguinte conjunto:

$$
X=\left\{T^{a}\left(t_{0}, \ldots, t_{n-1}\right) \mid T^{a} \in T_{a}, a=\left\langle a_{0}, \ldots, a_{n-1}\right\rangle \in \tau, t_{k} \in T_{k}\right\} .
$$

Como se pode ver nessa definição, $a \neq 0,1, \ldots, n-1$. A idéia intuitiva por trás desta restrição é que não formamos fórmulas atômicas apenas com termos individuais.

Seja $s=\left\{N, C,(\Pi x) \mid x \in V_{a}, a \in \tau\right\}$, tal que $N=\langle 0\rangle, C=\langle 0,0\rangle$, e $(\Pi x)=\langle 0\rangle$. Os conectivos e quantificadores são operações construídas a partir dos correspondentes tipos da sequência $s$; e, para nos acomodarmos à notação usual, na álgebra livre (que existe e é única, pelo teorema acima) denotaremos a operação correspondente ao tipo $N$ por $\neg$, a operação binária $C$ por $\rightarrow$, e os operadores unários $\Pi x$ por $\forall x$. $P(V, R)$ é a $s$-álgebra livre sobre o conjunto $X$ acima, com $V$ e $R$ como acima definidos.

Um elemento $w$ de $P(V, R)$ é chamado fórmula. 
Definição 3.8 Seja $w \in P(V, R)$, o conjunto $V(w)$ é o conjunto de variáveis da fórmula $w$, assim definido:

$$
V(w)=\bigcap\{U \mid U \subseteq V, w \in P(U, R)\} .
$$

As variáveis que ocorrem numa fórmula podem ser livres ou ligadas. Definimos o conjunto das variáveis livres da seguinte maneira:

Definição 3.9 Seja $w \in P(V, R)$. O conjunto var $(w)$ de variáveis livres de $w$ é definido por indução:

1. $\operatorname{var}\left(R^{a}\left(t_{0}, \ldots, t_{n-1}\right)\right)=\left\{t_{i}: t_{i} \in V_{i}\right\}$;

2. $\operatorname{var}\left(X^{a}\left(t_{0}, \ldots, t_{n-1}\right)\right)=\left\{t_{i}: t_{i} \in V_{i}\right\} \cup\left\{X^{a}\right\}$;

3. $\operatorname{var}(\neg w)=\operatorname{var}(w)$;

4. $\operatorname{var}\left(w_{1} \rightarrow w_{2}\right)=\operatorname{var}\left(w_{1} \cup \operatorname{var}\left(w_{2}\right)\right.$;

5. $\operatorname{var}(\forall x w)=\operatorname{var}(w)-\{x\}$.

Isso conclui nossa construção das linguagens formais como álgebras livres. Temos a linguagem da teoria simples de tipos, e um aparato dedutivo pode ser fornecido ao se separar algumas fórmulas para atuarem como axiomas e ao se especificarem regras de inferência (para detalhes sobre como isto pode ser feito, o leitor pode consultar o acessível trabalho de Copi 1971).

Podemos agora considerar fragmentos da linguagem da teoria de tipos acima exposta. Mais abaixo, mostraremos como podemos obter aquelas que são conhecidas como linguagens de primeira, segunda ou n-ésima ordem. Consideremos a seguinte definição:

Definição 3.10 A ordem de um termo é a ordem de seu tipo.

Para obtermos linguagens de segunda ordem, por exemplo, temos de nos restringir a termos cujos tipos sejam de ordem 1 ou menos, e, em particular, a variáveis de ordem 1 ou menos. Além disso, temos que considerar, ao construir o tipo de similaridade $s$, somente quantificadores sobre variáveis de tipos disponíveis a nós, ou seja, nesse caso, àquelas cujas ordens são 1 ou menos. Assim, quantificaremos somente sobre indivíduos ou propriedades e relações sobre indivíduos. Da mesma maneira, podemos obter linguagens de n-ésima ordem restringindo o conjunto de termos à ordem $n-1$ ou menos, e, da mesma forma, permitindo quantificação apenas sobre variáveis de ordem $n-1$ ou menos. Resumimos esse raciocínio na seguinte definição:

Principia 14(1): 15-30 (2010). 
Definição 3.11 A ordem de uma linguagem $£$ é definida como

$$
\operatorname{ord}(£)=\max \left\{\operatorname{ord}\left(x_{a}\right): x_{a} \in V_{a}\right\}+1 \text {. }
$$

Esta definição, em nossa opinião, capta a noção intuitiva de que a ordem de uma linguagem nos informa sobre os conjuntos sobre os quais quantificamos, ou, mais precisamente, sobre a ordem dos objetos sobre os quais quantificamos.

\section{Linguagem e estruturas}

Nesta seção, relacionamos os dois desenvolvimentos feitos acima: a teoria de estruturas e linguagens vistas como álgebras livres. Associada a cada estrutura $e$ podemos construir uma linguagem formal que nos permitirá falar dos elementos desta estrutura, linguagem esta que terá como símbolos de constante exatamente um símbolo para cada relação presente na estrutura, com ambos, o símbolo de constante e a correspondente relação, sendo de mesmo tipo. Nossa proposta é a de que dada uma estrutura $e$, para construir uma linguagem adequada a essa estrutura devemos impor que a ordem dos termos da linguagem sejam iguais ou menores que a ordem da estrutura. ${ }^{2}$ Dessa forma, o conjunto $X$ de livres geradores da álgebra livre, como definido acima, será restrito a esses termos, isto é, ao construirmos uma linguagem formal como uma álgebra livre, o conjunto de livres geradores será constituído pelas fórmulas atômicas construídas através dos símbolos disponíveis.

Como consequência dessa discussão, as linguagens nas quais podemos tratar de modo mais natural dos elementos de uma estrutura são as linguagens $£$ tais que $\operatorname{ord}(e) \leq \operatorname{ord}(£)$. Por exemplo, para estruturas de primeira ordem é conveniente usar, no mínimo, linguagens de primeira ordem. Tomemos, como um exemplo simples, a teoria de grupos, que lida com grupos (estruturas de primeira ordem). Essas estruturas, pela abordagem aqui desenvolvida, são tratadas de forma mais natural por linguagens de segunda ordem, pois assim podemos falar sobre subgrupos e quantificar sobre subconjuntos do domínio. Isso, naturalmente, não significa que seja impossível usar linguagens de primeira ordem nesse caso. Na realidade, a linguagem usualmente utilizada para desenvolver a teoria de grupos é uma linguagem de primeira ordem, a linguagem da teoria de conjuntos ZFC, que também é usada para tratar de outras teorias que não são de primeira ordem, como estruturas bemordenadas e corpos de Dedekind completos (Kunen 2009). Enfatizando mais uma vez, não estamos negando que se possa estudar estruturas de ordem $m$ utilizando uma linguagem de ordem menor que $m$, por exemplo, para se verificar que tipo de resultados podem ser obtidos em uma linguagem assim limitada, ou o quanto da teoria pode ser assim desenvolvida; estamos apenas afirmando que nossa definição

Principia 14(1): 15-30 (2010). 
permite estabelecer de certo modo as linguagens que nos permitem tratar de modo mais natural das estruturas em questão.

Apresentada uma linguagem apropriada (no sentido acima discutido) para se falar dos elementos de uma estrutura e, é relativamente simples definir, para essa linguagem, a noção de uma estrutura satisfazendo uma sentença $\alpha$ dessa linguagem no sentido tarskiano, isto é, $e=\alpha$, bem como a noção de consequência semântica $\Gamma \models \alpha$. Não apresentaremos estas definições aqui, mas note que no caso de estruturas de ordem superior restringimos nossa apresentação a estruturas principais, ou seja, as chamadas estruturas secundárias, ou estruturas de Henkin, não serão considerados por nós.

\section{Predicado de Suppes e abordagem semântica}

Dada uma estrutura e uma linguagem adequada para essa estrutura, discutiremos nessa seção como formular o predicado de Suppes a partir dessa estrutura usando essa linguagem (seguiremos da Costa e Chuaqui 1988). Nesta seção, vamos expor os desenvolvimentos técnicos do trabalho de da Costa e Chuaqui, que deram uma formulação precisa da idéia suppesiana de que axiomatizar uma teoria é dar-lhe um predicado conjuntista.

Recordemos, inicialmente, a definição de tipo de similaridade de uma estrutura $e$ : informalmente falando, trata-se de uma família de tipos que determina o tipo das relações presentes na estrutura. De acordo com essa definição, duas estruturas têm o mesmo tipo de similaridade se os tipos de suas relações formam a mesma família, e se as estruturas têm o mesmo número de conjuntos em seus domínios.

Sejam $e=\left\langle D_{n}, R_{\iota}\right\rangle$ e $g=\left\langle E_{n}, L_{l}\right\rangle$ estruturas de mesmo tipo de similaridade. Podemos estender uma dada função $f: D_{k} \mapsto E_{k}$ com $k<n$ para uma função de $\varepsilon\left(D_{n}\right)$ em $\varepsilon\left(E_{n}\right)$ do seguinte modo:

Definição 5.1 Seja $f$ uma função como a descrita acima, para cada tipo a $\in$ definimos:

1. Para os objetos de tipo $k$, com $0 \leq k<n$, temos $\left.f\left(D_{k}\right)=\left\{f(x): x \in D_{k}\right)\right\}$;

2. Para $a \in \tau$ tal que $a=\left\langle a_{0}, \ldots, a_{n-1}\right\rangle$, e $R$ o conjunto de objetos de tipo tipo $a$, temos $f(R)=\mathscr{P}\left(f\left(t_{a_{0}}\right) \times f\left(t_{a_{1}}\right) \times \ldots \times f\left(t_{a_{n-1}}\right)\right)$

Essa função associa objetos de tipo $a$ em $\varepsilon\left(D_{n}\right)$ a objetos de tipo $a$ em $\varepsilon\left(E_{n}\right)$. O caso mais interessante é quando a seguinte definição é verificada:

Definição 5.2 Sejam e $=\left\langle D_{n}, R_{\iota}\right\rangle$ e $g=\left\langle E_{n}, L_{\iota}\right\rangle$ estruturas de mesmo tipo de similaridade $s_{\lambda<\iota}$, e seja $f$ uma função bijetora de $D_{k}$ em $E_{k}$ com $0 \leq k<n$, dizemos que 
a família $f^{\prime}=f_{s_{\lambda<\iota}}$ é um isomorfismo entre e e $g$ quando $f_{a}\left(R^{a}\right)=L^{a}$, onde $R^{a}$ e $L^{a}$ significam que $R$ e L têm tipo a.

Definição 5.3 Uma sentença $\Phi$ da linguagem apropriada para a estrutura e é dita transportável se para toda estrutura $g$ isomorfa a e temos que

$$
e \models \Phi \Leftrightarrow g \mid=\Phi .
$$

Agora, com estas noções em mãos, podemos formular a definição de predicado de Suppes:

Definição 5.4 Um predicado de Suppes é uma fórmula $P(e)$ da linguagem da teoria de conjuntos que diz que e é uma estrutura de tipo de similaridade s satisfazendo $\Gamma$, um conjunto de sentenças transportáveis $\Phi$ de uma linguagem adequada a e.

Quando ocorre $\mathrm{P}(e)$, isto é, quando $e$ satisfaz $\mathrm{P}$, e é dita uma P-estrutura. De acordo com da Costa e Chuaqui (da Costa, Chuaqui 1988, p. 104), essa definição captura o sentido no qual podemos dizer que uma teoria é uma classe de modelos, precisamente a classe de modelos que são P-estruturas para algum predicado $\mathrm{P}$ adequado. Nesse sentido, a formulação do predicado de Suppes aqui apresentada pode ser vista como uma formulação extremamente rigorosa da afirmação informal de que, para os defensores da abordagem semântica, uma teoria é uma classe de modelos.

Há um ponto importante a ser notado aqui: podemos tratar, pelo menos em princípio, de todas as teorias científicas deste modo, fornecendo um predicado de Suppes para axiomatizar a teoria, com uma exceção. Considere a teoria que estamos utilizando para escrever os predicados de Suppes: a teoria de conjuntos ZFC. Não podemos fornecer um predicado de Suppes para ZFC do mesmo modo que faríamos para outras teorias científicas, como a mecânica de partículas. Este ponto fica claro se considerarmos que ZFC não pode fornecer modelos para si mesmo, de acordo com o segundo teorema de incompletude de Gödel. Ou seja, se assumirmos que ZFC é consistente, temos que os modelos de ZFC não serão modelos no sentido tarskiano usual, construídos dentro da teoria de estruturas apresentada acima. Este ponto, pouco enfatizado em geral, revela o grande poder da linguagem da teoria dos conjuntos e seu grande poder unificador.

Outro ponto a ser notado é que os modelos que caracterizam uma teoria, como definido acima, são realmente modelos no sentido lógico, da definição tarskiana usual. Apresentamos uma linguagem e uma estrutura que deve satisfazer axiomas formulados nesta linguagem. Como estivemos assumindo uma forma de interpretação canônica, em que cada símbolo não-lógico da linguagem é atribuído a uma 
relação correspondente na estrutura, foi possível deixar implícita a noção de interpretação dos símbolos não lógicos que está sempre envolvida na definição usual de modelos.

Por outro lado, poderíamos ter abordado o problema por outro viés. Ao invés de termos selecionado uma linguagem formal que fizemos corresponder à estruturas particulares, poderíamos ter simplesmente escrito os axiomas no predicado de Suppes diretamente na linguagem da teoria de conjuntos. Neste caso, não estaria envolvida uma definição de satisfação entre estrutura e linguagem, como é requerido pelos modelos na definição usual mas, antes, provaríamos que as relações que figuram na estrutura em questão possuem as propriedades exigidas pelo predicado de Suppes. Para ilustrar a situação com um exemplo, consideremos o modo como a aritmética de Peano é desenvolvida nos textos usuais de teoria de conjuntos (ver Enderton 1977). Provamos que em ZFC existe uma estrutura $\langle\omega, 0, \sigma\rangle$, algumas vezes chamada de estrutura de Peano, em que $\omega$ é o conjunto dos ordinais finitos de von Neumann, 0 é o conjunto vazio que, por definição, pertence a $\omega$, e $\sigma$ é a função sucessor que, para cada $n \in \omega$, atribui $n \cup\{n\}$, o seu sucessor por definição. Provamos que os axiomas de Peano são "satisfeitos" diretamente em ZFC; por exemplo, que $\sigma$ é injetora e que $\omega$ é indutivo. Assim, os axiomas de Peano são teoremas de ZFC quando entendidos como propriedades desta estrutura particular. Nesse caso, não está envolvida uma linguagem específica da aritmética que deve ser interpretada nesta estrutura, a fim de mostrar que a estrutura satisfaz os axiomas formulados nesta linguagem. Já quando apresentamos uma linguagem para a aritmética, por exemplo, de primeira ordem $\epsilon_{A R}=\{0,+, \cdot\}$, e a interpretamos na estrutura de Peano em ZFC, precisamos de uma atribuição de objetos na estrutura para os símbolos da linguagem, freqüentemente realizada por uma função interpretação, e de relações de satisfação entre sequências de elementos do domínio e fórmulas escritas nesta linguagem. Em particular, para as fórmulas que representam a versão em primeira ordem dos axiomas de Peano, teremos que na interpretação usual a estrutura de Peano será modelo, no sentido de Tarski, da aritmética de Peano quando esta for formulada em linguagem de primeira ordem.

Desta discussão, temos que existem dois sentidos diferentes em que uma teoria pode ser uma classe de modelos. No primeiro sentido, a palavra modelo é usada de modo um pouco diverso daquele empregado nos textos de lógica usuais; no segundo, conforme estamos empregando neste trabalho, o sentido da palavra modelo é o usual. Este é um ponto pouco discutido na literatura sobre a abordagem semântica. Uma questão que podemos colocar é se na primeira abordagem, quando escrevemos os axiomas diretamente na linguagem da teoria de conjuntos, teremos que o predicado de Suppes realmente determina as mesmas classes de estruturas que são obtidas quando formulamos os axiomas de uma teoria numa linguagem formal específica, ou se há classes que podem ser obtidas por uma abordagem, mas 
não por outra.Não entraremos nestas discussões aqui, mas o leitor interessado pode consultar Marshall e Chuaqui 1991.

Consideremos agora exemplos de predicados de Suppes para a teoria de grupos, e com menos detalhes para os corpos reais e espaços vetoriais sobre este corpo.

Exemplo 5.1 O predicado de Suppes para a teoria de grupos

Seja $G$ um conjunto; como definido previamente, introduzimos a função $t_{G}$, ou simplesmente $t$, cujo domínio é o conjunto $\tau$ de tipos, para criar a escala $\varepsilon(G)$. Escolhemos, então, a relação o de tipo $\langle 0,0,0\rangle$, isto é, $\circ \in \mathscr{P}(G \times G \times G)$, uma relação - de tipo $\langle 0,0\rangle$, isto é, $-\in \mathscr{P}(G \times G)$ e um elemento $i$ de tipo 0 , isto é, $i \in G$. De acordo com as definições estabelecidas anteriormente, a ordem de cada uma das relações é 1 e a ordem de $i$ é 0 . Lembrando o fato de que uma função $n$-ária é uma relação $n+1$-ária, a operação usual de composição se torna uma relação ternária, e a operação inverso se torna uma relação binária.

A estrutura de grupo é $\mathfrak{G}=\langle G, \circ,-, i\rangle$ e a ordem dessa estrutura é a maior ordem de suas relações, logo, ord(G) é 1 . O próximo passo é escrever os postulados e dar o predicado conjuntista.

Como definido anteriormente, a linguagem para $\mathfrak{G}$ é uma linguagem de segunda ordem. O conjunto $T$ de termos é formado por um conjunto de variáveis $V=\bigcup V_{a}$, tal que $\operatorname{ord}(a) \leq 1$ e o conjunto $\left\{\mathrm{o}^{\langle 0,0,0\rangle},-^{\langle 0,0\rangle}, i^{0}\right\}$ de símbolos de constante. $\mathrm{O}$ tipo de similaridade $s$ para essa linguagem é $s=\left\{\neg, \rightarrow,(\forall x) \mid x \in V_{a}, a \in \tau\right\}$, com a restrição feita acima de que $\operatorname{ord}(a) \leq 1$. Para o conjunto $X$ de livres geradores tomamos $X=\left\{T^{a}\left(t_{0}, \ldots, t_{n-1}\right) \mid T^{a} \in T_{a}, a=\left\langle a_{0}, \ldots, a_{n-1}\right\rangle \in \tau, t_{k} \in T_{k}\right\}$, portanto, pelo teorema 3.1 existe uma álgebra livre sobre o conjunto de geradores $X$, e essa é a linguagem para a estrutura $\mathfrak{G}$.

Com a linguagem especificada, podemos escrever os axiomas usuais para a teoria de grupos. Denominando-os A1, A2 e A3, escrevemos:

1. $\forall x \forall y \forall z(((x \circ y) \circ z)=(x \circ(y \circ z)))$

2. $\forall x \exists y(x-y=i)$

3. $\forall x(x \circ i=x)$

Então, o predicado de Suppes para a teoria de grupos é escrito da seguinte maneira:

$$
\begin{aligned}
\mathfrak{G}(X) \Longleftrightarrow & \exists G \exists \circ \exists-\exists i(X=\langle G, \circ,-, i\rangle \wedge(\circ \in t(\langle 0,0,0\rangle)) \\
& \wedge(-\in t(\langle 0,0\rangle)) \wedge(i \in t(0)) \wedge A 1 \wedge A 2 \wedge A 3) .
\end{aligned}
$$

Passamos agora aos exemplos do predicado para corpos e espaços vetoriais reais. Não examinaremos com todos os detalhes. 
Exemplo 5.2 O predicado de Suppes para corpos e espaços vetoriais

Inicialmente, vamos apresentar o predicado de Suppes para corpos. Nesta estrutura há apenas um conjunto base, o conjunto $K$. Os objetos desse conjunto são de tipo 0 . As operações são $+, \cdot, 0,1,<$, que são de tipos $\langle 0,0,0\rangle,\langle 0,0,0\rangle, 0,0$ e $\langle 0,0\rangle$, respectivamente. Fazemos um pequeno alerta aqui para que o leitor não confunda o símbolo de tipo 0 com o elemento 0 corpo. De maneira usual, essas são as operações de adição, multiplicação, o elemento neutro da adição, o elemento neutro da multiplicação e a relação "menor que" entre os elementos de $K$. A linguagem dos corpos, de acordo com a nossa abordagem, é uma linguagem de segunda ordem language. Os axiomas para corpos reais são bem conhecidos e são fórmulas transportáveis; denotaremos por $C_{i}$ a conjunção dos axiomas de corpo no seu predicado de Suppes:

$$
\begin{aligned}
\mathfrak{K}(X) \Longleftrightarrow & \exists K \exists+\exists \cdot \exists 0 \exists 1 \exists<(X=\langle K,+, \cdot, 0,1,<\rangle \wedge(+\in t(\langle 0,0,0\rangle)) \\
& \left.\wedge(\cdot \in t(\langle 0,0,0\rangle)) \wedge(0 \in t(0)) \wedge(1 \in t(0)) \wedge(<\in t(\langle 0,0\rangle)) \wedge C_{i}\right) .
\end{aligned}
$$

Com auxílio da noção de corpo, podemos apresentar a estrutura de espaço vetorial $\mathfrak{V}$ sobre este corpo. Nesse estrutura há dois conjuntos base, o conjunto $V$ de vetores e o conjunto $K$ de escalares. Os objetos de $V$ são do tipo 1 . Junto com as operações do corpo, teremos aqui as operações $\overline{+}, \overline{-}, \overline{0}$ que são de tipos $\langle 1,1,1\rangle,\langle 0,1,1\rangle$, 1 , respectivamente. Um espaço vetorial euclidiano é um espaço vetorial com a adição de produto interno e produto vetorial, denotados por $(\mathbf{x}, \mathbf{y})$ e $[\mathbf{x}, \mathbf{y}]$ e cujos tipos são $\langle 1,1,0\rangle$ e $\langle 1,1,1\rangle$, respectivamente. As ordens das linguagens de espaço vetorial e de espaço vetorial euclidiano são, de acordo com nossa abordagem, 2, isto é, uma linguagem de segunda ordem. Os axiomas para essas estruturas são bem conhecidos e claramente transportáveis; denotaremos a conjunção dos axiomas específicos para espaço vetorial por $V_{i}$. O predicado de Suppes pode ser escrito como:

$$
\begin{aligned}
\mathfrak{V}(X) \Longleftrightarrow & \exists K \exists V \exists+\exists \cdot \exists 0 \exists 1 \exists<\exists \bar{\mp} \exists \cdot \exists \overline{0}(X=\langle V, K,+, \cdot, 0,1,<, \bar{\mp}, \bar{\cdot}, \overline{0}\rangle \\
& \wedge(+\in t(\langle 0,0,0\rangle)) \wedge(\cdot \in t(\langle 0,0,0\rangle)) \wedge(0 \in t(0)) \\
& \wedge(1 \in t(0)) \wedge(<\in t(\langle 0,0\rangle)) \wedge(\bar{\mp} \in t(\langle 1,1,1\rangle)) \\
& \left.\wedge(\bar{\bullet} \in t(\langle 0,1,1\rangle)) \wedge(\overline{0} \in t(1)) \wedge C_{i}\right) .
\end{aligned}
$$

\section{Conclusão}

A contribuição que buscamos dar nesse artigo foi expor, inspirados nos trabalhos de Newton da Costa, uma abordagem unificada do conceito de estrutura e de uma linguagem associada a uma estrutura. Estamos cientes de certas complicações e tecnicismos envolvidos em nossa exposição; no entanto, o rigor daí provindo nos parece

Principia 14(1): 15-30 (2010). 
de inegável valor em qualquer discussão que trate dos fundamentos (no caso deste artigo) da concepção semântica de teorias científicas. Ademais, a abordagem aqui desenvolvida nos parece bastante instigante quando temos em vista os importantes resultados que podem e estão sendo obtidos a partir dela. A título do exemplo, podemos citar a teoria de Galois generalizada desenvolvida por Newton da Costa e Alexandre Rodrigues 2007. Por fim, nos parece claro que ainda que discussões e desenvolvimentos num nível mais informal sejam muito frutíferas e motivadoras, certos tópicos da filosofia da ciência ganhariam muito se a discussão se desse num nível mais formal e rigoroso como, por exemplo, através das ferramentas aqui propostas.

\section{Referências}

Barnes, D. W. \& Mack, J. M. 1975. An Algebraic Introduction to Mathematical Logic. New York:Springer-Verlag.

Bourbaki, N. 1948. The Architecture of Mathematics. The American Mathematical Monthly 57(4): 221-32.

- 1968. Theory of Sets. Paris: Hermann and Addison-Wesley.

Copi, I. M. 1971. The Theory of Logical Types. London:Routledge \& Kegan Paul .

da Costa, N. C. A. \& Chuaqui, R. 1988. On Suppes' Set Theoretical Predicates. Erkenntnis 29: 95-112.

da Costa, N. C. A. \& Rodrigues, A. A. M. 2007. Definability and Invariance. Studia Logica 82: $1-30$.

Enderton, H. B. 1977. Elements of Set Theory. New York:Academic Press.

Kunen, K. 2009. The Foundations of Mathematics. London:College Publications.

Marshall, M. V. \& Chuaqui, R. 1991. Sentences of Type Theory: the only sentences preserved under isomorphism. The Journal of Symbolic Logic 56(3): 932-48.

Suppe, F. 1977. The search for philosophic understanding of scientific theories. In F. Suppe (Ed.) The structure of scientific theories. Urbana: University of Illinois Press, 6-232.

- 1989. The Semantic Conception of Theories and Scientific Realism. Chicago: University of Illinois Press, 3-20.

Uebel, T. 2006. Vienna Circle, Stanford Encyclopedia of Philosophy. http://plato.stanford.edu/entries/vienna-circle/, acessado em 22/08/2010.

Jonas R. Becker Arenhart and Fernando T. F. Moraes Programa de Pós-Graduação em Filosofia Universidade Federal de Santa Catarina Florianópolis, SC Brasil jonas. becker2@gmail.com fermatadeu@uol.com.br

Principia 14(1): 15-30 (2010). 
Resumo. Neste artigo, a partir de tópicos presentes na obra de Newton C. A. da Costa, propomos uma fundamentação rigorosa para de uma possível formulação de teorias científicas através da abordagem semântica. Seguindo da Costa, primeiramente desenvolveremos uma teoria geral das estruturas; no contexto desta teoria de estruturas mostraremos como caracterizar linguagens formais como um tipo particular de estrutura, mais especificamente, como uma álgebra livre. Em seguida, discutiremos como associar uma linguagem a uma estrutura, com a qual poderemos formular axiomas que buscam captar a teoria da estrutura. Por fim, mostraremos como podemos, utilizando este aparato conceitual, fundamentar a formalização de da Costa e Chuaqui do chamado predicado de Suppes, utilizado para caracterizar teorias científicas de modo rigoroso.

Palavras-chave: Abordagem semântica, estruturas, modelos, predicado de Suppes.

\section{Notes}

${ }^{1}$ Recentemente, esta visão de aparente consenso entre os membros do círculo tem sido questionada, mostrando que havia pelo menos duas correntes predominantes entre os membros do grupo (ver Uebel 2006).

${ }^{2}$ Seguimos aqui uma sugestão de da Costa.

Principia 14(1): 15-30 (2010). 